65 $5^{\text {ème }}$ Congrès de la SFCO, 03029 (2017)

DOI: $10.1051 / \mathrm{sfco} / 20176503029$

\title{
POSTER
}

\section{A propos d'une lésion pigmentée de la fibro-muqueuse palatine.}

\section{Bohbot A ${ }^{1}$, Alantar $A^{2}$, Zerat $L^{3}$, Dame $\mathbf{M}^{2}$, Pauphilet $C^{4}$}

1. Faculté de Médecine, Université Paris Descartes -Université Paris $V$

2. Unité fonctionnelle de Chirurgie Orale - Hôpital Max Fourestier - Nanterre

3. Laboratoire Lavergne

4. Service de Dermatologie - Hôpital Max Fourestier - Nanterre

Un patient de 45 ans est reçu en consultation de chirurgie orale en décembre 2016. II n'a pas d'antécédents médico-chirurgical ni allergie. L'examen exo-buccal est normal. L'examen endo-buccal met en évidence une lésion palatine symétrique pigmentée ancienne asymptomatique sur fond érythémateux, épargnant la ligne médiane antérieure avec des pétéchies de part et d'autre. La compression à l'aide d'un manche de miroir entraîne un blanchiment de la lésion. Le patient a pris du Lariam® (dérivé de la quinine) régulièrement avant de partir à Madagascar en 2010/2014/2015, en prévention du paludisme. L'hypothèse d'une coloration iatrogène induite par le Lariam® a été privilégiée (Soria et coll., 2015; Martinelli-Kläy et coll., 2012). Toutefois la compression à l'origine d'un blanchiment peut être en faveur d'une lésion vasculaire. Une biopsie d'emblée est dans ce cas un geste dangereux et imprudent sans avoir écarté au préalable un trouble de l'hémostase par un bilan sanguin (hémogramme, plaquette, TP, TCA) et une malformation artério-veineuse par une IRM. Les bilans sanguin et IRM étant négatifs, une biopsie est réalisée et conclue à un « angiome caverneux avec dépôt ferriques associés». La coloration PAS est négative. Devant un diagnostic aussi exceptionnel au palais une deuxième lecture anatomopathologique est demandée. Cette dernière retient une pigmentation ferrique (colorations de Fontana négative et de Pearls positive). L'absence de disposition nodulaire des vaisseaux sanguins confirmée par immunomarquage élimine un angiome II n'y aucun signe de dysplasie ni lésions vasculaires pré-existantes. Aucun signe fonctionnel ni doléance esthétique n'étant rapportés par le patient, la lésion est surveillée annuellement. Ce cas souligne l'importance de la biopsie dans l'étiquetage des lésions buccales atypiques en particulier pigmentaires palatines permettant d'indiquer une prise en charge adaptée.

bohbotanthony@gmail.com

(C) The authors, published by EDP Sciences. This is an Open Access article distributed under the terms of the Creative Commons Attribution License 4.0 (http://creativecommons.org/licenses/by/4.0/) 